\title{
So, what is a Primer?
}

We look back on the first 5 years of the journal and share where we hope to go next.

\section{$\mathbb{C}$ there is more we can do to be inclusive of patients in our journal gy}

When we launched Nature Reviews Disease Primers 5 years ago, our marketing campaign put forward a dictionary-type definition to answer the question 'What is a Primer?' Primer, $n$. - An introductory review article covering one disease or disorder - that describes global epidemiology, molecular mechanisms, diagnosis and management. The vision for the journal began by thinking about what early career researchers or medical students entering a field might need to know and thinking about how experts would signpost the achievements of the field - something of a 'state of the nation address', but with the Nature Reviews treatment. To achieve this goal, we aimed to recruit senior researchers from the realms of basic science and the clinic and task them with telling readers what is most important to know. Now, 5 years later, we have put this concept to good use, resulting in the publication of 225 Primers on a diverse range of medical topics, from nonalcoholic fatty liver disease $^{1}$ to malaria ${ }^{2}$ and opioid use disorder ${ }^{3}$. As we have interacted intensively with the medical communities we serve, it is increasingly clear that we can do more for readers.

Patients are, and should be, at the very heart of what we do, and we are committed to providing information that can be useful to them. This approach, by way of our PrimeView visual summaries, has been welcomed. Through feedback from the communities, we understand that patients can be vocal self-advocates who help researchers focus their programmes to address the issues that affect them the most. This is most evident in the rare-disease space, where patients have long been asked for their input in agenda setting. But there is more we can do to be inclusive of patients in our journal content.

We recently started asking authors to capture the patient perspective in their Primers. What does this look like in practice? In some cases, we included direct quotes from patients about how their conditions affect them ${ }^{4}$. In others, patient advocates were involved in the writing of the Primer ${ }^{5}$. Of course, it would be unreasonable to assume that the few individuals who are included in the Primer are representative of the whole patient population. Our reason for asking authors to include these perspectives is to give readers a glimpse into what reality might be like for these individuals. We also think that these viewpoints can offer insight for medical students, who we consider a key part of our audience.
Our efforts to extend inclusivity reach further than involving patients in the writing process, and we are constantly evolving our approach. We have long felt that representation of different global regions (where diagnostic and treatment practices might vary) in our articles is important. Additionally, almost all our Primers (95\%) have at least one woman in the author panel and $23 \%$ have been led by a female researcher. We clearly still have work to do to push these boundaries. We also think that it is vitally important to present diversity in terms of age, ethnicity, body type and hair texture when we illustrate people in our articles, in particular in the patient-focused PrimeViews. In line with our principles, we have pledged support to end the stigma of obesity by avoiding ${ }^{6}$, for example, stigmatizing language and imagery when describing people with obesity. We are now also providing captions for individuals who use screen reader software on our Twitter account (@DiseasePrimers).

Primers take a long time to produce - some take years - because of the number of people involved, and the 'trademark' structure and length of the manuscripts. Combined with the aim for Primers to be detailed introductions to a topic, covering certain subjects, such as the COVID-19 pandemic, in this format is not always feasible or appropriate. Accordingly, we have decided to explore publishing shorter articles, in which authors can discuss timely topics and highlight challenges and opportunities faced across medical specialties and of global relevance. To get us started, today, we publish five Comments. As a nod to our anniversary, we asked five past authors to reflect on the Primers they have led, developments in their fields and our editorial processes. These are presented alongside the corresponding original Primers and PrimeViews in an online collection.

We also aim for readers to hear more from us by publishing Editorials when we have something important to share. Please also continue to get in touch with us $@$ @iseasePrimers or at future conferences.

1. Brunt, E. M. et al. Nonalcoholic fatty liver disease. Nat. Rev. Dis. Primers 1, 15080 (2015).

2. Phillips, M. A. et al. Malaria. Nat. Rev. Dis. Primers 3, 17050 (2017).

3. Strang, J. et al. Opioid use disorder. Nat. Rev. Dis. Primers 6, 3 (2020).

4. Sabat, R. et al. Hidradenitis suppurativa. Nat. Rev. Dis. Primers 6, 18 (2020)

5. Lord, C. et al. Autism spectrum disorder. Nat. Rev. Dis. Primers 6, 5 (2020).

6. Rubino, F. et al. Joint international consensus statement for ending stigma of obesity. Nat. Med. 26, 485-497 (2020). 